\title{
The dynamic response of carbon fiber-filled polymer composites
}

\author{
D.M. Dattelbaum ${ }^{1}$, R.L. Gustavsen ${ }^{1}$, S.A. Sheffield ${ }^{1}$, D.B. Stahl ${ }^{1}$, R.J. Scharff ${ }^{1}$, P.A. Rigg ${ }^{1}$, \\ J. Furmanski ${ }^{2}$, E.B. Orler ${ }^{2}$, B. Patterson ${ }^{2}$, and J.D. Coe $^{3}$ \\ ${ }^{1}$ Weapons Experiments Division, Los Alamos National Laboratory, Los Alamos, NM 87545, USA \\ 2 Materials Science and Technology Division, Los Alamos National Laboratory, Los Alamos, NM 87545, USA \\ 3 Theoretical Division, Los Alamos National Laboratory, Los Alamos, NM 87545, USA
}

\begin{abstract}
The dynamic (shock) responses of two carbon fiber-filled polymer composites have been quantified using gas gun-driven plate impact experimentation. The first composite is a filament-wound, highly unidirectional carbon fiber-filled epoxy with a high degree of porosity. The second composite is a chopped carbon fiber- and graphite-filled phenolic resin with littleto-no porosity. Hugoniot data are presented for the carbon fiber-epoxy (CE) composite to $18.6 \mathrm{GPa}$ in the through-thickness direction, in which the shock propagates normal to the fibers. The data are best represented by a linear Rankine-Hugoniot fit: $U_{s}=2.87+1.17 \times u_{p}\left(\rho_{0}=1.536 \mathrm{~g} / \mathrm{cm}^{3}\right)$. The shock wave structures were found to be highly heterogeneous, both due to the anisotropic nature of the fiber-epoxy microstructure, and the high degree of void volume. Plate impact experiments were also performed on a carbon fiber-filled phenolic (CP) composite to much higher shock input pressures, exceeding the reactants-toproducts transition common to polymers. The $\mathrm{CP}$ was found to be stiffer than the filament-wound $\mathrm{CE}$ in the unreacted Hugoniot regime, and transformed to products near the shock-driven reaction threshold on the principal Hugoniot previously shown for the phenolic binder itself. [19] On-going research is focused on interrogating the direction-dependent dyanamic response and dynamic failure strength (spall) for the CE composite in the TT and $0^{\circ}$ (fiber) directions.
\end{abstract}

\section{Introduction}

Graphite- or carbon fiber-loaded polymer composites have been used extensively in the automotive and aerospace industries because they combine high strength and low fatigue properties, with a lighter weight than most metals and alloys, and low cost. The mechanical response of carbon fiber-based composites is known to vary substantially with variations in composition, including fiber content, degree of porosity, type of epoxy binder and polymer-filler adhesion, and other microstructural details [1-3]. Despite their widespread application, there are few reports detailing their impact (shock) and dynamic strength properties, typically limited to low shock pressures [4-9]. There have also been investigations of the dynamic response of related (such as glass- or alumina-filled) polymer composites [10-13].

We have performed shock compression experiments on two types of carbon fiber-filled polymeric composites to quantify and compare their dynamic responses. The first composite is a filament-wound, unidirectional carbon-fiber epoxy composite with a high degree of porosity $(\sim 16 \%)$. The second composite is a chopped carbon fiber-filled phenolic resin containing $\sim 8 \%$ graphite powder, and littleto-no porosity. Here, we present shock Hugoniot data of the unidirectional composite in the through-thickness (TT) direction, in which the shock propagates normal to the fiber direction up to $18.6 \mathrm{GPa}$. Hugoniot data are also presented for a carbon-filled phenolic resin at much higher pressures up to nearly $50 \mathrm{GPa}$, well past the reactants-to-products transition driven by shock compression and heating on the principal Hugoniot.

\section{Experimental}

The results of plate impact experiments on two types of carbon-filled polymeric composites are described. Table 1 summarizes the compositions of the two composites.
Table 1. Description of materials studied.

\begin{tabular}{|l|c|c|}
\hline Sample & $\begin{array}{c}\text { Initial density } \\
\left(\mathbf{g} / \mathbf{c m}^{3}\right.\end{array}$ & Composition (by wt) \\
\hline $\begin{array}{l}\text { Carbon fiber- } \\
\text { filled epoxy }\end{array}$ & $\begin{array}{c}1.536 \text { (skeletal) } \\
1.314 \text { (bulk) }\end{array}$ & $\begin{array}{c}60-64 \% \text { HexTow } \\
\text { carbon fibers } \\
36-40 \% \text { 55A epoxy }\end{array}$ \\
\hline $\begin{array}{l}\text { Carbon fiber } \\
\text { filled phenolic }\end{array}$ & 1.555 & $\begin{array}{c}\sim 56.4 \% \text { PAN-based } \\
\text { carbon fibers } \\
\end{array}$ \\
& & $\begin{array}{c}7.8 \% \text { graphite powder } \\
35 \% \text { phenolic resin }\end{array}$ \\
\hline
\end{tabular}

\subsection{Materials}

\subsubsection{Carbon fiber-filled epoxy (CE)}

The unidirectional carbon fiber-epoxy composite was prepared by filament winding on a mandrel at $90^{\circ}$ (hoop), allowing for partial cure, then flattening the material into sheets to form a unidirectional B-staged laminate. Several laminate layers were then stacked and condensed in a hot press to form the panels used in this study. The composite consists of HexTow ${ }^{\text {TM }}$ IM7 carbon fibers embedded in a "55A" epoxy resin $[14,15]$. The 55A epoxy is an 80:20 blend of the diglycidyl ether of bisphenol A (DGEBA), and the diglycidyl ether of 1,4-butanediol, which is cured (or hardened) with a 60:40 mixture of methylene dianiline (MDA) and m-phenylene diamine ( $\mathrm{m}$ PDA) (Tonex 60/40) [14]. Scanning electron micrographs of the composite are shown in Figure 1 at 3 magnifications. The HexTow ${ }^{\mathrm{TM}}$ fibers are $\sim 5 \mu \mathrm{m}$ in diameter, and the fiber alignment is exceptionally unidirectional. By thermogravimetric analysis, the composite contains 60-64 wt\% carbon fibers, $x \%$ epoxy resin and, in contrast to related composites, a large amount of porosity $(\sim 16 \%)$ in the form of voids that run unidirectionally through the entire sample thickness, as seen in Figure 1 (top). The sound velocities of the bulk composite in the TT direction illustrate the 


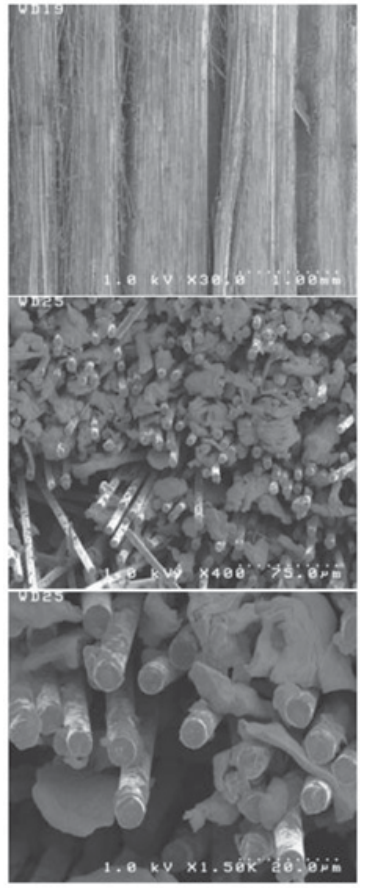

Fig. 1. Scanning electron micrographs of the graphite-epoxy composite at 3 magnifications. From the micrographs, the high degree of porosity is evident.

anisotropic nature of the composite: $c_{l}=2.54 \mathrm{~km} / \mathrm{s}, c_{s}=$ $1.34 \mathrm{~km} / \mathrm{s}[1]$.

\subsubsection{Carbon fiber-filled phenolic (CP)}

The second composite studied is a chopped carbon fiberand graphite powder-filled phenolic composite with an initial density of $1.55 \mathrm{~g} / \mathrm{cm}^{3}$. The polyacrylonitrile (PAN)based carbon fibers are $99+\%$ carbon and $6.5 \mu$ m diameter. The final composite is black in color with visible "swirls" of the non-oriented chopped carbon fibers in the resin. There was no evidence of porosity in the CP composite.

\subsection{Plate impact experiments}

Well-defined shock conditions were imparted to composite samples using plate impact experiments driven by light gas and powder guns within the Shock and Detonation Physics group at LANL. These include a gas-driven single stage gun $(78 \mathrm{~mm})$, a two-stage light gas gun $(50 \mathrm{~mm})$, and a high performance powder gun $(28 \mathrm{~mm})$. The respective launch tube diameters are given in parentheses. The projectile velocity range of the collective gun capabilities spans from $<0.1$ to $\sim 8 \mathrm{~km} / \mathrm{s}$.

Two types of plate impact experiments were performed. In reverse ballistic or front surface impact (FSI) experiments, the composite sample (approx. $6.35 \mathrm{~mm}$ thick) was mounted in the front of a polycarbonate projectile, and impacted into a window material used as an equation-ofstate standard, such as oriented single-crystal LiF. The particle velocity at the impact interface, measured using dual velocity interferometers (VISARs) [16], combined with the measured projectile velocity were used to determine the Hugoniot state using impedance matching methods.

The second type of experiment is referred to as a "top-hat" geometry and is used to make shock wave transmission ( $\mathrm{T})$ measurements. In this configuration, a material used as an EOS standard, in this case oxygenfree high-purity Copper (OFHC), is impacted into a drive plate of the same material. The drive plate is backed by a sample of the composite, and a rear window (LiF). The shock transit time is measured through the sample using photon Doppler velocimetry (PDV) [17] and/or VISAR, each at three positions - two on the rear surface of the drive plate and one on the rear windowed-interface of the sample. Shock wave profiles at the sample-LiF interface are also obtained in the experiment following shock transit through $\sim 3 \mathrm{~mm}$ or $\sim 6 \mathrm{~mm}$ of composite. Projectile velocity is measured using either a single PDV probe collimated down the gun launch tube, or by the sequential cut-off of 4 laser diodes by the projectile at the launch tube exit aperture.

\section{Results}

\subsection{Carbon fiber-filled epoxy (CE)}

Five front surface impact experiments were performed on the carbon fiber-epoxy (CE) composite, and the results are summarized in Table 2 and plotted in Figure 3. The Hugoniot data derived from the FSI experiments define the principal Hugoniot in the TT direction from 2.5 to 18.6 GPa. Figure 2 shows a representative interface particle velocity wave profile from shot $2 \mathrm{~s}-360$, in which the composite was impacted into $\mathrm{LiF}$ at $2.16 \mathrm{~km} / \mathrm{s}$. In the FSI configuration, the high density surface of the sample impacts the LiF window, resulting in a transient, high interfacial particle velocity followed by a decrease in particle velocity and complex, heterogeneous profile. In some of the experiments, evidence of a "knee" in the front of the wave profile was also observed. Both features are due to the highly complex microstructure of the composite with a thin epoxy film on the surfaces, and large degree of void volume behind the high density surface. Millett et al. observed similar, but less pronounced structure in their recorded shock wave profiles in the TT direction using manganin gauges $[4,5]$. The Hugoniot loci reported in Table 2 are derived from the peak particle velocity at the interface and are therefore representative of the high density $\left(\rho=1.536 \mathrm{~g} / \mathrm{cm}^{3}\right)$ surface of the composite. Using an average particle velocity across the heterogeneous wave structure after the initial drop at the interface, combined with the bulk density using impedance matching methods results in Hugoniot loci that differ by those reported in Table 1 by $\sim 3-6 \%$, which are lower in calculated shock pressure.

In addition to the FSI experiments, a single transmission experiment was performed on the composite in the TT direction, using embedded electromagnetic gauges [18] to measure both the shock and particle velocities independently. In this experiment, the CE sample was sandwiched between two electromagnetic gauge elements, each 
Table 2. Summary of Hugoniot data of a carbon fiber-epoxy composite in the through-thickness (TT) direction.

\begin{tabular}{|c|c|c|c|c|c|c|c|c|}
\hline $\begin{array}{c}\text { Shot } \\
\text { number }\end{array}$ & $\begin{array}{c}\text { Experiment } \\
\text { type }^{\mathrm{a}}\end{array}$ & $\begin{array}{c}\text { Initial } \\
\text { density } \\
\mathrm{g} / \mathrm{cm}^{3}\end{array}$ & $\begin{array}{c}\text { Density } \\
\left(\mathrm{skeletal}^{3}\right. \\
\mathrm{g} / \mathrm{cm}^{3}\end{array}$ & $\begin{array}{c}\text { Impactor or } \\
\text { window }^{\mathrm{a}}\end{array}$ & $\begin{array}{c}\text { Projectile } \\
\text { velocity } \\
(\mathrm{km} / \mathrm{s})\end{array}$ & $\begin{array}{c}u_{p} \\
(\mathrm{~mm} / \mu \mathrm{s})\end{array}$ & $\begin{array}{c}U_{s} \\
(\mathrm{~mm} / \mu \mathrm{s})\end{array}$ & $\begin{array}{c}\text { Pressure } \\
(\mathrm{GPa})\end{array}$ \\
\hline $1 \mathrm{~s}-1388$ & FSI & 1.314 & 1.536 & $\mathrm{LiF}$ & 0.826 & 0.594 & 3.68 & 3.4 \\
\hline $1 \mathrm{~s}-1389$ & FSI & 1.349 & 1.536 & $\mathrm{LiF}$ & 0.670 & 0.491 & 3.37 & 2.5 \\
\hline $2 \mathrm{~s}-360$ & FSI & 1.377 & 1.536 & $\mathrm{LiF}$ & 2.163 & 1.509 & 4.49 & 10.4 \\
\hline $2 \mathrm{~s}-386$ & FSI & 1.402 & 1.536 & $\mathrm{LiF}$ & 2.592 & 1.765 & 5.04 & 13.7 \\
\hline 2s-387 & FSI & 1.406 & 1.536 & $\mathrm{LiF}$ & 3.307 & 2.237 & 5.42 & 18.6 \\
\hline 2s-446 & $\mathrm{T}$ & 1.330 & - & Kel-F 81 & 3.055 & 1.750 & 4.97 & 11.6 \\
\hline
\end{tabular}

${ }^{a}$ In FSI experiments, the window material that the sample is impacted into is listed. In $2 s-446$, the composite was impacted directly by a Kel-F 81 polymer impactor.

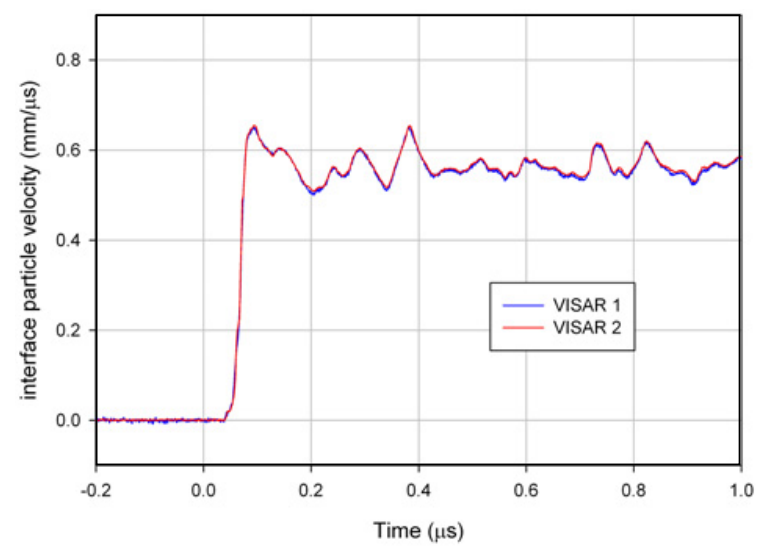

Fig. 2. Interface particle velocity profile recorded using dual VISARs from shot 2s-360, in which the carbon fiber-epoxy composite impacted $\mathrm{LiF}$ at $2.163 \mathrm{~km} / \mathrm{s}$ resulting in a shock pressure of $10.4 \mathrm{GPa}$ in the composite.

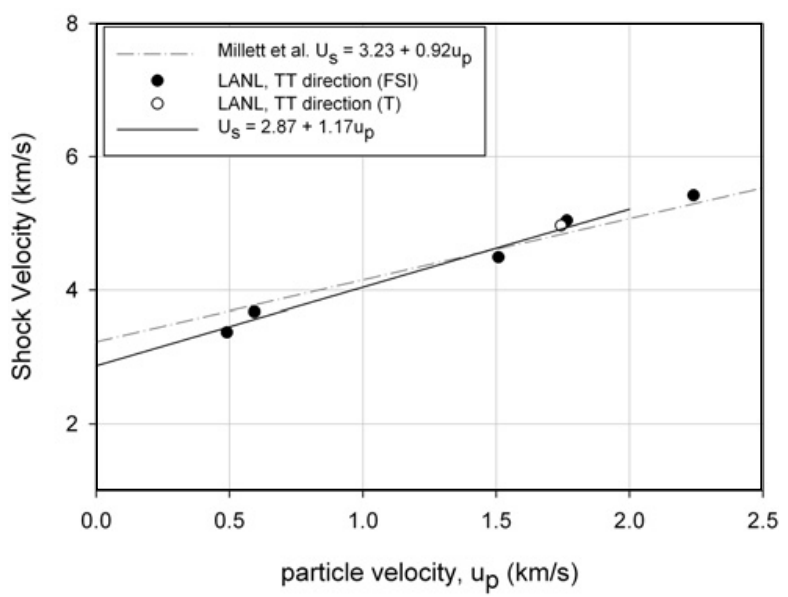

Fig. 3. Hugoniot data for $\mathrm{CE}$ in the shock velocity particle velocity plane from FSI and T experiments. The linear RankineHugoniot fit to the FSI data is also shown as the solid line, along with Millet's fit to Hugoniot data for a related composite.

consisting of $5 \mu \mathrm{m} \mathrm{Al}$ contained in FEP-Teflon, forming a membrane $25 \mu \mathrm{m}$ thick. The target was then placed in a magnetic field $(1.2 \mathrm{kG})$ at the end of the launch tube. The particle velocity was determined directly from the response of the electromagnetic gauge, with a voltage rise proportional to $u_{p}$. The shock velocity was determined by the shock transit time through the composite sample. In the $\mathrm{T}$ experiment, shot $2 \mathrm{~s}-446$, the bulk density $(\rho=$ $\left.1.330 \mathrm{~g} / \mathrm{cm}^{3}\right)$ is interrogated, and the resulting Hugoniot point is summarized in Table 2, and plotted in Figure 3. The shock wave profile at the impact interface was also found to be heterogeneous, and an average $\mathrm{u}_{\mathrm{p}}$ is reported.

The Hugoniot data for the CE material in the TT direction are shown in the shock velocity-particle velocity plane in Figure 3 (black circles = FSI experiments). The locus determined from the $\mathrm{T}$ experiment is also shown as the open symbol. A linear Rankine-Hugoniot fit to the TT data from the FSI experiments to $10.4 \mathrm{GPa}$ is $U_{s}=2.87+$ $1.17 \times u_{p}\left(\rho_{0}=1.536 \mathrm{~g} / \mathrm{cm}^{3}\right)$, shown in Figure 3 as the solid line. Shock-driven reaction would not be expected below shock input pressures of $\sim 20 \mathrm{GPa}$, if the composite was solid density. Note that Carter and Marsh did not observe reaction in neat epoxy until $23.1 \mathrm{GPa}$, or a volumetric compression of $\mathrm{V} / \mathrm{V}_{0}=0.597$ [19]. Here, we fit the unreacted Hugoniot data to $10.4 \mathrm{GPa}$, since we cannot rule out shockinduced reaction at higher pressures due to the large degree of porosity in the CE. Including the high-pressure locus at $18.6 \mathrm{GPa}$ changes the linear fit only slightly, $U_{s}=2.89+$ $1.14 \times u_{p}$ and suggests that the CE remains unreacted to 18.6 GPa. Furthermore, we expect, as in all polymers, that the extrapolation of the Rankine-Hugoniot fit to $u_{p}=0$ is inappropriate, due to anticipated curvature at low $u_{p}$ from free volume and viscoelastic effects. The reported data define the high density, unreacted Hugoniot for a carbonfiber epoxy composite in the TT direction.

\subsection{Carbon fiber-filled phenolic (CP)}

A number of plate impact experiments were also performed on the carbon fiber-filled phenolic in FSI and T geometries resulting in the Hugoniot data summarized in Table 3. The shock pressures reached in the $\mathrm{CP}$ are significantly higher than those in the CE material, and range from $\sim 10.0 \mathrm{GPa}$ to nearly $50 \mathrm{GPa}$. A representative particle velocity wave profile from shot 69ts-10-14, the highest-pressure shot, is shown in Figure 4. In contrast to the CE composite, the shock profile is flat, and there are no signs of precursor waves or heterogeneity in the wave structure.

Hugoniot data for $\mathrm{CP}$ are plotted in Figure 5 in the shock velocity-particle velocity plane. Carter and Marsh previously reported Hugoniot data for an unfiilled phenolic resin, the binder used in the CP composite [19]. Their Hugoniot data for the neat phenolic are also shown in Fig. 5 for comparison. A clear cusp, or non-linearity in the neat phenolic data is observed between $\mathrm{u}_{\mathrm{p}}=$ $2.5-3.0 \mathrm{~km} / \mathrm{s}$. Similar to most polymers, phenolic 
Table 3. Summary of Hugoniot data on the CP composite. Error in the measured projectile velocities and Hugoniot variables is $\leq 2 \%$.

\begin{tabular}{|c|c|c|c|c|c|c|c|}
\hline $\begin{array}{c}\text { Shot } \\
\text { number }\end{array}$ & $\begin{array}{c}\text { Experiment }^{\text {type }} \\
\text { ty }^{\mathrm{a}}\end{array}$ & $\begin{array}{c}\text { Initial } \\
\text { density } \\
\left(\mathrm{g} / \mathrm{cm}^{3}\right) \\
\pm 0.001\end{array}$ & $\begin{array}{c}\text { Impactor or } \\
\text { window }^{\mathrm{c}}\end{array}$ & $\begin{array}{c}\text { Projectile } \\
\text { velocity } \\
(\mathrm{km} / \mathrm{s})\end{array}$ & $u_{p}(\mathrm{~km} / \mathrm{s})$ & $U_{s}(\mathrm{~km} / \mathrm{s})$ & $\begin{array}{c}\text { Pressure } \\
(\mathrm{GPa})\end{array}$ \\
\hline $2 \mathrm{~s}-423$ & FSI & 1.556 & $\mathrm{LiF}$ & 2.962 & 1.940 & 5.795 & 17.6 \\
\hline $2 \mathrm{~s}-435$ & FSI & 1.555 & LiF & 3.340 & 2.188 & 6.171 & 20.9 \\
\hline $2 \mathrm{~s}-456$ & $\mathrm{~T}$ & 1.554 & OFHC & 3.176 & 2.576 & 6.462 & 25.9 \\
\hline $2 \mathrm{~s}-572$ & FSI & 1.550 & LiF & 1.993 & 1.363 & 4.720 & 10.0 \\
\hline 69 ts-10-09 & $\mathrm{T}$ & 1.554 & OFHC & 3.686 & 2.977 & 6.818 & 31.5 \\
\hline 69 ts-10-14 & $\mathrm{T}$ & 1.556 & OFHC & 4.919 & 3.947 & 7.614 & 46.8 \\
\hline 69 ts-11-02 & $\mathrm{T}$ & 1.555 & OFHC & 4.002 & 3.238 & 6.886 & 34.7 \\
\hline 69 ts-11-04 & $\mathrm{T}$ & 1.556 & OFHC & 4.567 & 3.752 & 7.314 & 42.7 \\
\hline
\end{tabular}

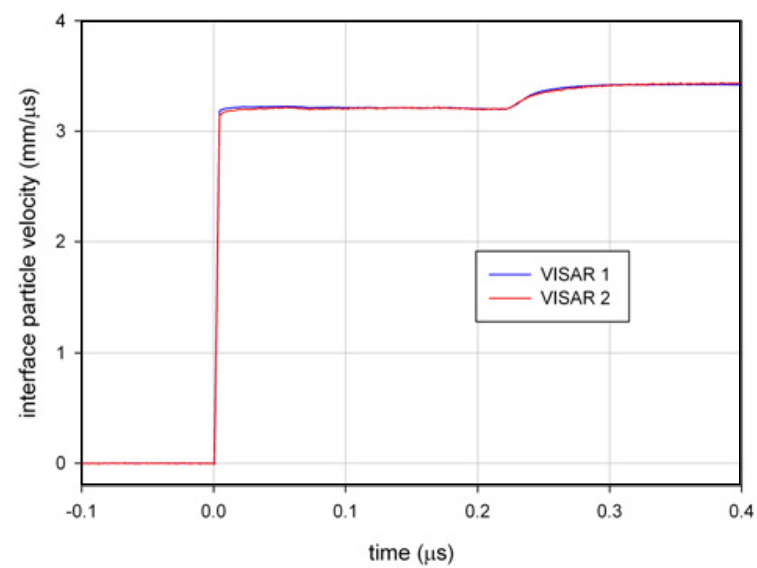

Fig. 4. Shock wave profile measured at the carbon-filled phenolic (CP)-LiF interface in shot 69 ts-10-14, in which the CP was shocked to $46.8 \mathrm{GPa}$. There is slight evidence of rounding in the front of the profile. The second wave is from the shock reflection from the $\mathrm{Cu}$ baseplate.

undergoes shock-induced reaction above $23 \mathrm{GPa}$ [19]. A cusp is also observed near this threshold $(25 \mathrm{GPa})$ in the composite, due to shock-driven chemical reaction, which is also clearly seen in the $\mathrm{P}-\mathrm{V} / \mathrm{V}_{0}$ plot in Figure 6 . A linear Rankine-Hugoniot fit to the 3 lowest pressure Hugoniot points is $U_{s}=2.309+1.777 \times u_{p}$, which is representative of the unreacted Hugoniot. In our experiments, the shock velocity was determined from the transit time of the first wave through the material, consistent with Carter and Marsh's experiments, and no attempt to perform multiwave analysis above the reaction threshold was made. However, in the cusp regime $(25-30 \mathrm{GPa})$, there is increased rounding in the front of the shock wave, which is a manifestation of chemical reaction occurring just behind the shock front. At $\sim 32 \mathrm{GPa}$, the transformation occurs over $<90 \mathrm{~ns}$ by the $2^{\text {nd }}$ wave rise time. Above $40 \mathrm{GPa}$, the shock wave sharpens again, as seen in Figure 4, consistent with rapid chemistry at these pressures. On-going work is developing a products equation of state for this material, and indicates the carbon transforms to diamond in this pressure regime [20].

\section{Discussion}

New plate impact experiments have been performed to quantify the Hugoniot-based equations-of-state for two

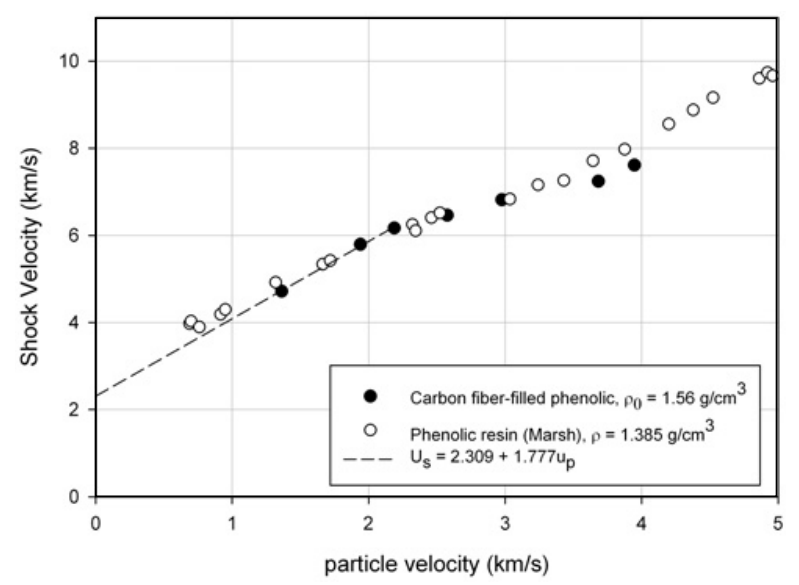

Fig. 5. Hugoniot data for CP overlaid with Marsh's data for a neat phenolic resin in the $U_{\mathrm{s}}-u_{\mathrm{p}}$ plane. The linear Rankine-Hugoniot fit to $20.9 \mathrm{GPa}$ is also shown and is representative of the unreacted EOS.

carbon-filled polymer composites. Filament-wound carbon fiber-epoxy composites are highly anisotropic, and their dynamic response must be characterized, at a minimum, in directions parallel and perpendicular to the fiber direction. Here, we have described the shock properties of a CE composite in the TT direction, in which the shock runs perpendicular (normal) to the fiber direction. The CE composite studied was highly porous, and contained a more ductile epoxy than related CE composites. These microstructural and chemical differences are manifested in the unreacted Hugoniot.

Millett et al. previously reported Hugoniot data for a similar carbon fiber-epoxy composite in the same direction to lower shock input pressures. In their work, the composite also had a lower initial density $\left(\rho_{0}=1.50 \mathrm{~g} / \mathrm{cm}^{3}\right)$, and the linear Rankine-Hugoniot fit to $u_{p} \sim 0.9 \mathrm{~km} / \mathrm{s}$ was reported to be $U_{s}=3.23+0.92 \times u_{p}[4,5]$. This linear fit (extrapolated to greater $u_{p}$ ) is shown in Figure 3 overlaid with the Hugoniot data on the CE composite studied here. Overall, the two near-full density Hugoniots in the TT direction are quite similar, with our recent work extending the shock input pressure regime interrogated. The greater slope of the Rankine-Hugoniot fit for the composite studied here may indicate that the pressure-dependence of the bulk modulus is greater, e.g. it is more compressible. This may be a manifestation of the greater ductility of the $55 \mathrm{~A}$ 


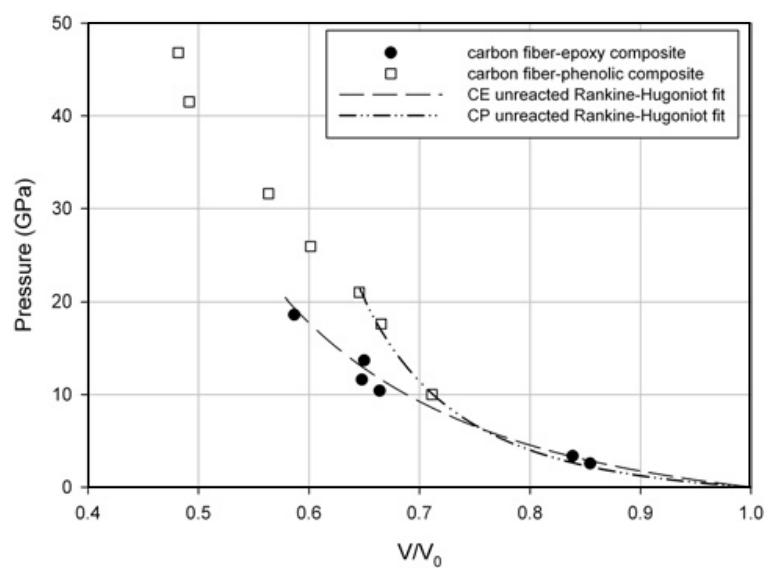

Fig. 6. Hugoniot data for $\mathrm{CE}$ and $\mathrm{CP}$ in $\mathrm{P}-\mathrm{V} / \mathrm{V}_{0}$ illustrating the greater compressibility of CE compared with the fully-dense CP. Rankine-Hugoniot fits defining the unreacted Hugoniots are also shown for the two materials to $\sim 18.6 \mathrm{GPa}$ for $\mathrm{CE}$ and $20.9 \mathrm{GPa}$ for $\mathrm{CP}$.

resin compared with more common epoxy resins, such as the Epon class of resins or, presumably, the Hexcel epoxy used in Millett's formulation. The linear fit to our data is also consistent with Dandekar's work on a glass fiberreinforced composite [13].

On-going work is focused on understanding the influence of anisotropy on the dynamic compression properties, and determination of dynamic (spall) strength of the CE composite in both directions. Preliminary results have shown that a pronounced quasi-elastic wave transmitted through the fibers [21] is evident in the CE composite shocked in the fiber $\left(0^{\circ}\right)$ direction, with wave velocities exceeding $10 \mathrm{~km} / \mathrm{s}$ at impact velocities $>2 \mathrm{~km} / \mathrm{s}$. In addition, we have found that the spall strength of the composite is weak ( $<1 \mathrm{GPa})$ in the TT direction, consistent with measurements of the quasi-static tensile strength for related composites [22].

The Hugoniot of a fully-dense carbon fiber-filled phenolic composite was also determined, through the shockdriven reactants-to-products transition. A comparison of the two carbon-filled polymer composites can be gained from the overlaid Hugoniot data in Figure 6. The full density $\mathrm{CP}$ composite is measurably stiffer in this plane, providing a representation of the dynamic response of high integrity, carbon filled composites. The CE undergoes greater compaction at the same shock pressure, which is likely a consequence of the ductile binder (55A epoxy), and porosity in the microstructure. Shock-induced reaction occurs in $\mathrm{CP}$ near the shock pressure threshold of the phenolic binder, previously characterized by Carter and Marsh [19]. Above $40 \mathrm{GPa}$, the Hugoniot data are best described by a products equation of state, with carbon represented thermochemically as diamond. It should be noted that the $\mathrm{CE}$ is expected to undergo similar shockinduced reactions at greater shock input conditions.

\section{Acknowledgements}

We acknowledge funding from DOE/NNSA and DOE/DoD Joint Munitions Program for this work. Los Alamos National Laboratory is operated by LANS LLC for the Department of
Energy. We also thank Brian Bartram, Adam Pacheco, Lee Gibson and Ben Hollowell for help in firing the gas guns at Chamber 9, Rob Hixson for technical discussions, and M. Sam Shaw for help with product equation of state development.

\section{References}

1. E. N. Brown, P. J. Rae, D. M. Dattelbaum, D. Stahl, Soc. Exp. Mech.- SEM Annual Conf. and Expos. On Exp. and Appl. Mech. 2010, 2, 1660.

2. H. M. Hsiao, I. M. Daniel, Composites: Part B 1998 29, 521.

3. A. Gilat, R. K. Goldberg, G. D. Roberts, Compos. Sci. Technol. 2002, 62, 1469.

4. J. C. F. Millett, N. K. Bourne, Y. J. E. Meziere, R. Vignjevic, A. Lukyanov Comp. Sci Tech. 2007, 67, 3253-3260.

5. R. Vignjevic, J. C. F. Millett, N. K. Bourne, Y. Meziere, A. Lukyanov Shock Compression of Condensed Matter - 2005, AIP Proceedings, 2006, pg. 825.

6. P. J. Hazell, C. Stennett, G. Cooper Composites: Part A 2009, 40, 204.

7. P. J. Hazell et al. Composites: Part A 2008, 39, 866. P. J. Hazell et al. Int. J. Imp. Eng. 2009, 36, 1136.

8. S. A. Bordzilovsky, et al. Shock Compression of Consensed Matter-1997, AIP Press, 1998, pg. 545.

9. T. H. Antoun, L. Seaman, D. R. Curran, "Dynamic failure of materials," Defense Special Weapons Agency Report DSWA-TR-96-77-V1.

10. E. Zaretsky, G. deBotton, M. Perl Int. J. Solids Struct. 2004, 41, 569.

11. D. E. Munson, R. R. Boade, K. W. Schuler J. Appl. Phys. 1978, 49, 4797.

12. R. E. Setchell, M. U. Anderson J. Appl. Phys. 2005, 97, 083518.

13. D. P. Dandekar, C. A. Hall, L. C. Chhabildas, W. D. Reinhart Comp. Struc. 2003, 61, 51-9.

14. M. A. Golub, N. R. Lerner, M. S. Hsu; "Kinetic Study of Polymerization/Curing of Filament-Wound Composite Epoxy Resin Systems with Aromatic Diamines," J. Appl. Polym. Sci., 32, 5215 (1986).

15. Hexcel HexTow ${ }^{\mathrm{TM}}$ Carbon Fiber datasheet.

16. L.M. Barker, R.E. Hollenbach, J. Appl. Phys. 41, 4208-4226 (1970).

17. O.T. Strand, D.R. Goosman, C. Martinez, T.L. Whitworth and W.W. Kuhlow, Rev. Sci. Inst. 77, 083108 (2006).

18. R.R. Alcon, S.A. Sheffield, A.R. Martinez, R.L. Gustavsen, Shock Compression of Condensed Matter1997, S.C. Schmidt, D.P. Dandekar, J.W. Forbes (Eds.), AIP Conference Proceedings 429, p. 845 (1997).

19. W.J. Carter, S.P. Marsh, Hugoniot equation of state of polymers, Los Alamos National Laboratory report LA-13006-MS (1995).

20. J. D. Coe, M. S. Shaw, unpublished results.

21. P.-L. Hereil et al. J. Phys. IV 1997, 7, 529.

22. Lin, J.-S. Polymers $\mathcal{F}$ Polymer Composites, 2000, 8, 107. 